\title{
Objective Assessment of Sunburn and Minimal Erythema Doses: Comparison of Noninvasive In Vivo Measuring Techniques after UVB Irradiation
}

\author{
Min-Wei Huang, ${ }^{1,2}$ Pei-Yu Lo, ${ }^{2}$ and Kuo-Sheng Cheng ${ }^{2}$ \\ ${ }^{1}$ Department of Psychiatry, Chia-Yi Veterans Hospital, Chia-Yi 600, Taiwan \\ ${ }^{2}$ Institute of Biomedical Engineering, National Cheng Kung University, Tainan 701, Taiwan
}

Correspondence should be addressed to Kuo-Sheng Cheng, kscheng@mail.ncku.edu.tw

Received 29 November 2009; Revised 9 February 2010; Accepted 30 March 2010

Academic Editor: Yingzi Du

Copyright (C) 2010 Min-Wei Huang et al. This is an open access article distributed under the Creative Commons Attribution License, which permits unrestricted use, distribution, and reproduction in any medium, provided the original work is properly cited.

\begin{abstract}
Military personnel movement is exposed to solar radiation and sunburn is a major problem which can cause lost workdays and lead to disciplinary action. This study was designed to identify correlation parameters in evaluating in vivo doses and epidermis changes following sunburn inflammation. Several noninvasive bioengineering techniques have made objective evaluations possible. The volar forearms of healthy volunteers $(n=20), 2$ areas, $20 \mathrm{~mm}$ in diameter, were irradiated with UVB $100 \mathrm{mj} / \mathrm{cm}^{2}$ and $200 \mathrm{mj} / \mathrm{cm}^{2}$, respectively. The skin changes were recorded by several monitored techniques before and 24 hours after UV exposures. Our results showed that chromameter $a^{*}$ value provides more reliable information and can be adopted with mathematical model in predicting the minimal erythema dose (MED) which showed lower than visual assessment by $10 \mathrm{mj} / \mathrm{cm}^{2}$ (Pearson correlation coefficient $\mathfrak{I}=0.758$ ). A more objective measure for evaluation of MED was established for photosensitive subjects' prediction and sunburn risks prevention.
\end{abstract}

\section{Introduction}

The main effect of UVB (wavelengths of 320 to $340 \mathrm{~nm}$ ) is thought to take place mainly in the epidermis. UVB is very active in human skin and can induce sunburn, tanning, and many photodermatoses after exposure. Both skin cancer and aging may occur following chronic repeated exposure [1]. Sunburn inflammation has been used as end point for many photobiologic studies of skin. The patient's minimal erythema dose (MED), defined as the minimal dose in producing just-perceptible erythema determined 24 hours after irradiation, is an example. Photosensitive subjects have low MED values and are vulnerable to UV radiation [2]. The sensitivity of human skin to UV radiation must be determined, especially military personnel movement that is exposed to sun, and sunburn is a major problem which can cause lost workdays and lead to disciplinary action. The assessment of acute effect of epidermis after UVB exposure is rarely analyzed by noninvasive quantitative means. The traditional visual MED reading lacks accuracy, reproducibility, and quantification. This study was designed to identify the correlation parameter in evaluation of in-vivo doses and epidermal changes following UVB irradiation using noninvasive techniques, especially those showing very weak reactions. Using more objective method, the photosensitive soldiers could be screened to prevent from sunburn risk.

\section{Materials and Methods}

\subsection{Materials}

2.1.1. UVB Irradiation Source. UVB irradiation was administered using a light box (UV 3001, Waldmann, Germany) irradiation unit.

2.1.2. Colorimeter (CR). For quantitative measurement of skin color, we made use of a chromameter (Minolta CR-400, Osaka, Japan) [3]. It measures the erythema and skin color based on Commission International de I'eclairage $L^{*} a^{*} b^{*}$ color space. The $L^{*} a^{*} b^{*}$ color space method (CIELAB), developed in 1976, is the most frequently used to objectively assess colors [4]. In this system the $L^{*}$ coordinate correlates 
with the intensity of the reflected light (brightness) and the $a^{*}$ and $b^{*}$ coordinates are chromatic, covering the spectrum from red to green and from yellow to blue, respectively. The $a^{*}$ value is well recognized to linearly correlate with skin erythema [5].

2.1.3. Multiprobe Adapter. The multiprobe adapter (MPA) is a flexible, economic plug-in system to combine all skin measurement probes of Courage + Khazaka electronic $\mathrm{GmbH}$ [3], including tewameter for transepidermal water loss (TEWL), corneometer CM825 for skin moisture, and mexameter Hb MX18 for skin pigmentation and erythema [6].

(a) Mexameter $\mathrm{Hb}$ (MI, EI). The melanin and erythema indexes were evaluated by a mexameter $\mathrm{Hb}$ (MX-18, Courage an Khazaka, Cologne, Germany). The mexameter is equipped with LED (light emitting diode) light sources and a silicon diode detector for detecting reflected light from skin. The instrument measures the intensity of reflected green $(568 \mathrm{~nm})$, red $(660 \mathrm{~nm})$, and infrared $(880 \mathrm{~nm})$. The definitions of the melanin (MI) and erythema (EI) indexes calculated automatically by the mexameter are as follows $[5,7]$ :

Melanin index:

$$
\mathrm{MI}=\frac{500}{\log 5(\log \text { infrared }-\log \mathrm{I} \text { red })+500},
$$

Erythema index:

$$
\mathrm{EI}=500=\log 5(\log \mathrm{I} \text { red }-\log \mathrm{I} \text { green })+500 .
$$

(b) TEWL Measurement (TEWL). TEWL was measured using an evaporimeter (Tewameter, Courage an Khazaka, Cologne, Germany) on the arms of subjects before and after a single dose of UV-light radiation. In vitro measurement of TEWL provides a clear indication of the skin barrier integrity. All investigations were performed at $23-25^{\circ} \mathrm{C}$ and 40-60 relative humidity [6].

(c) Electrical Capacitance (COR). Electrical capacitance was measured with corneometer (CM 825, Courage an Khazaka, Cologne, Germany). The technique is based on the completely different dielectric constant of water and other substances (mostly $>7$ ). The measuring capacitor shows changes of capacitance according to the moisture content of the samples and provides temperature stability. The capacitor shows changes of capacitance according to moisture content of the samples [6].

2.1.4. Laser Scanning Confocal Microscopy (LSCM). The skin changes have been extensively investigated by histological examination. But, biopsy may alter the original morphology and induce an iatrogenic trauma, and thus noninvasive methods are more desirable for application. Laser scanning confocal microscopy (LSCM) allows noninvasive in-vivo optical sectioning of layers of skin in real time. Using melanin as main endogenous contrast, the technique can analyze the epidermis at a cellular level [8]. Since epidermis changes after UV irradiation are usually examined by biopsy, it is quite difficult to monitor the skin changes dynamically. Therefore, we aimed to test the potential of LSCM of epidermis in vivo after UVB irradiation [9].

A commercially available LSCM (Vivascope1500, Lucid, Henrietta, New York) was used [10]. The following parameters are assessed: thickness of stratum corneum (SC), measured from skin surface to the first recognizable nucleus in the granular layer; and minimal thickness of epidermis (DP), defined as the distance between the skin surface and the most apical recognizable dermal structure $[8,11]$.

2.1.5. Laser Doppler Perfusion Imager (LDI). Laser Doppler perfusion imager (LDI) is a standard technique in the noninvasive monitor of blood flow and has been widely applied in the studies of vascular changes within skin area of interest. In UV irradiation, dermal microperfusion is increased when inflammation induced by UV exposure starts with vasodilatation. In this study, cutaneous microcirculation was measured with a laser Doppler Perfusion imager (Moor LDI2, UK) [7]. The output of the LDI system consists of two different two-dimensional data sets, perfusion and total back-scattered light intensity (TLI), with a point-to-point correspondence. The blood perfusion data set, represented by a color-coded image, was calculated from back-scattered and Doppler-shifted light, defined as the product of red blood cells' mean velocity times their concentration in the sampled tissue volume. The second data set maps the TLI and was coded into a photographic-liked gray-scale image of the lesion [4]. The distance between laser Doppler perfusion imager and skin surface is $30 \mathrm{~cm}$ (with measurements taken from centre of the studied area for all subjects). In this study, each recorded image consists of multiple measurement sites and represents the blood perfusion in a skin area of approximately $68 \times 86 \mathrm{~mm}^{2}$.

\subsection{Methods}

2.2.1. Study Design and Subjects. This study was approved by the ethics committee for human studies of Veterans General Hospital-Kaohsiung. Twenty healthy Chinese volunteers (15 males and 5 females with mean age at $28 \mathrm{y} / \mathrm{o}$, SD 5.6), who gave their informed consents, were enrolled. Subjects have not been exposed to systemic corticosteroids, immunosuppresive medicines, or sunbathing in the past 4 weeks. Both temperature and humidity in the room were recorded. The temperature was maintained within the range between $20^{\circ} \mathrm{C}$ and $25^{\circ} \mathrm{C}$ and relative humidity was within $40 \%$ to $60 \%$. Smoking was not allowed within 4 hours prior to the measurements. Both coffee and tea intakes were not allowed within 1 hour prior to measurement. To design the study properly, the knowledge of intraregional variation and daily variability of measurement parameter is of utmost importance. In our study, the measurement for each individual was taken at exactly the same time each day. Each skin site acts as its own control in measurement of basal skin color and blood flow on the skin on day 1 [12]. 
2.2.2. Broadband Light Testing to UVB. Broadband light testing to UVB was conducted for all subjects as follows. The test sites are nonexposed skin of the mid-lower back. The exposure doses for the MED testing were $50 \mathrm{mj} / \mathrm{cm}^{2}, 70 \mathrm{mj} / \mathrm{cm}^{2}$, $100 \mathrm{mj} / \mathrm{cm}^{2}, \quad 120 \mathrm{mj} / \mathrm{cm}^{2}, \quad 140 \mathrm{mj} / \mathrm{cm}^{2}$, and $160 \mathrm{mj} / \mathrm{cm}^{2}$, respectively, according to previous experience [8]. These were determined visually by 2 experienced investigators. The readings of erythema were also taken for all exposure sites using chromameter. Digital photographs were taken.

\subsubsection{Traditional Visual MED Reading (VS) [7, 12]. There} are

0 No erythema,

+ Menimal perceptible erythema with sharp borders (1 MED),

+ Pink erythema,

++ Marked erythema, no edema, no pain,

+++ Fair red erythema, mild edema, mild pain,

++++ Violaceus erythema, marked edema, strong pain, strong edema, partial blistering.

2.2.4. Background Skin Reaction Measurements. The constitutional skin color was measured at infra-axillary areas with chromameter. The forearm of each individual was positioned on an arm support at heart level for 15 minutes. The baseline values of cutaneous blood flow and skin condition were measured for all test sites as described below.

2.2.5. The Test Standard Dosage of UVB Irradiation. The MED does not exhibit an incremental increase of erythema with increasing UV doses. Takiwaki et al. suggested that 2 independent MEDs are more appropriate to assess the UVinduced skin reactions in oriental skin since tanning induced by doses lower than 2 MEDs is too weak to discriminate the differences between various reactions. On the contrary, doses higher than 2 MEDs often result in desquamation, which makes assessment by colorimeter difficult [13]. The mean MED of all suspected photosensitive subjects from 2000 to 2005 in our hospital is $107.3 \pm 30.72 \mathrm{mj} / \mathrm{cm}^{2}(85$ individuals, 45 females and 40 males with a mean age at 50.3 years) from our previous studies. Accordingly, $100 \mathrm{mj} / \mathrm{cm}^{2}$ and $200 \mathrm{mj} / \mathrm{cm}^{2}$ were chosen as our test standard.

Forearm (flexor side) is used for monitoring of skin reactions. Each test area was marked using a template with 2 holes and a green ink pen. On the ventral side of right forearm (about 3-4 cm distance from the anticubitale fossa and the wrist), 2 areas of each volunteer with each $20 \mathrm{~mm}$ in diameter were irradiated with UVB $100 \mathrm{mj} / \mathrm{cm}^{2}$, and $200 \mathrm{mj} / \mathrm{cm}^{2}$ respectively. The skin of volar forearm skin before UV exposure and 24 hours later was examined by traditional visual MED reading (VS), tewameter (TEWL), mexameter (E, M), corneometer (COR), laser scanning confocal microscopy (LSCM) for SC and DP reading, chromameter (L, A, B), and laser-Doppler imager (LDI).

2.2.6. Image Processing and Data Analysis Method for Extraction of Perfusion Parameters of Laser-Doppler Imager. The proposed data analysis method consists of three concrete steps.

(a) Evaluation of data validity to exclude data having inherent artifacts: before processing of perfusion and TLI images, the collected material was preprocessed to identify the unwanted artifacts induced by involuntary patient movements. The preprocessing procedure involves visual inspection and comparison of multiple TLI images emanating from the same lesion.

(b) Lesion delineation to define the optical boundaries of each lesion in TLI imaging: the region of interest (ROI) was superimposed on the perfusion image, and the perfusion parameters are calculated.

(c) Blood perfusion feature extraction and resulting values were statistically evaluated between different irradiated groups.

2.3. Statistics. The time course of different measurements was analyzed within the context of repeated-measurement ANOVA models because measurements over time and over measurements are repeatedly taken within the same volunteers. A method can be considered to be discriminatory if the changes of skin condition were detected and ought to be judged significant. The discriminatory ability of different measurements was compared using respective $F$-values of the ANOVA models. The highest $F$-value represents the best discriminatory ability.

An ANOVA model was calculated for every combination of measurement. A dose-dependent effect of a specific measurement was significant if the $P$-value of the $F$-test is $<.05$. This corresponds to an $F$-value of 4.35 in a given situation (DF 1, 19).

In the second step, pairwise comparisons for the time dependent effect between $t 0$ and $t 24$ were performed in each ANOVA model. A time-dependent effect between two time points was classified as statistically signification if the $P$-value is $<.05$. This is true for a (Bonferroni-adjusted) $t$-value of 1.725 in the given situation (DF 19). All calculations were done using SPSS10.0.

\section{Results}

3.1. Constitutive Colorimetric Readings. The background readings before irradiation gave a mean of $58.6 \pm 2.9 \mathrm{~L}^{*}$ (L) units, $5.7 \pm 1.2 a^{*}(\mathrm{~A})$ units, and $13.6 \pm 1.2 b^{*}$ (B) units when read with chromameter, respectively. The value of erythema index (EI) was $271 \pm 66$ units and melanin index (MI) $216.7 \pm 44$ units using the mexamter Hb. No significant correlation was observed between MEDs and constitutive colorimetric readings.

3.2. Assessment of Melanin Pigmentation after UV Irradiation by Laser Scanning Confocal Microscopy. Laser scanning confocal microscopy (LSCM) was used to measure the melanocytes in forearm skin after ultraviolet exposure 


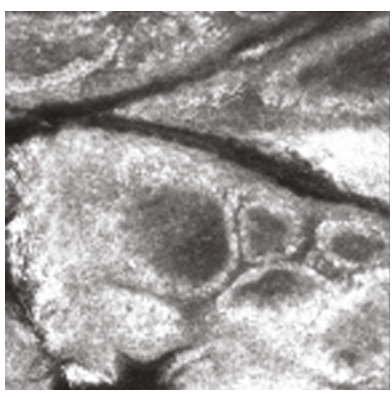

(a)

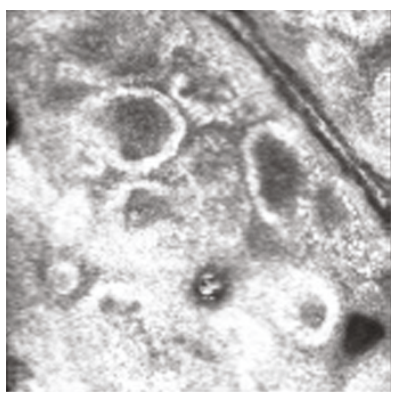

(c)

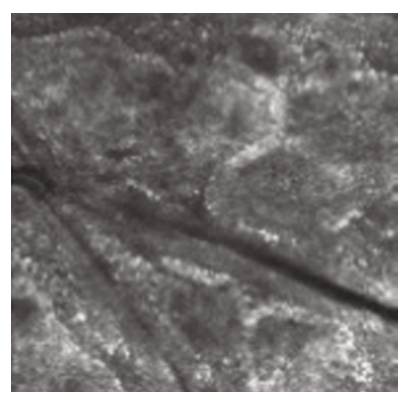

(b)

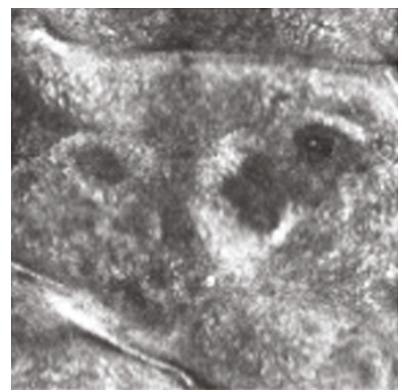

(d)
FIGURE 1: Laser scanning confocal microscopy (LSCM) of supranuclear melanin caps of dermal-epidermal junction in the forearm skin in relation to UVB exposure. (a) and (b) One representative case before and after (24 hours) $100 \mathrm{mj}$ UVB irradiation. (c) and (d) The same case before and after (24 hours) $200 \mathrm{mj}$ UVB irradiation.

(Figure 1). The brightness of basal layer in LSCM decreased 24 hours after UV irradiation exposure.

3.3. Assessment of Blood Flux after UVB Irradiation. The software of LDI can demonstrate both the flux image and video images. The LDI features a camera in production of color images of scanned area, making the positioning and comparison of images easier. Before UVB irradiation, we observed no increase of blood flow. Twenty four hours later after 100 or $200 \mathrm{mj} / \mathrm{cm}^{2}$ UVB exposure, the color scale of perfusion image increases (Figure 2). The blood perfusion feature was extracted and statistically evaluated between different irradiated areas.

\subsection{Statically Analysis of Different Noninvasive Biomedical} Techniques. Twenty volunteers are enrolled in all noninvasive biomedical analysis. The mean differences of measurements related to UV exposure are listed in Table 1. It is evident that EI, VS, and $a^{*}$ increase with higher UVB irradiation. On the contrary, $\mathrm{MI}, L^{*}$, and $b^{*}$ decrease. TEWL, COR, SC, DP, and LDI show discrepant. The repeated measure ANOVA test found a discrimination of MI and EI of mexameter, L, A, and B of chormameter, LDI and visual score (VS). Among them, MI, EI, A, and VS exhibited the hightest discrimination power. The pairwise comparison ( $t$-test) is significant for $100 \mathrm{mj} / \mathrm{cm}^{2}$ and $200 \mathrm{mj} / \mathrm{cm}^{2}$ UVB-irradiated doses in MI, EI, L, A, B, and VS. The LDI is only effective at
$200 \mathrm{mj} / \mathrm{cm}^{2}$-irradiated doses. There is no discrimination for SC, DP, COR, and TEWL.

In summary, both $a^{*}$ and erythema index (EI) show positive linear relation to VS. The Pearson correlation coefficients of $a^{*}$ value and EI relating visual scoring are compared $\left(P=.578\right.$ over .501 at $100 \mathrm{mj} / \mathrm{cm}^{2} ; P=.767$ over .759 at $200 \mathrm{mj} / \mathrm{cm}^{2}$ ). The $a^{*}$ value is better than EI index. The $a^{*}$ value provides more reliable information and can be used in mathematical model in predicting the minimal erythema dose (MED).

3.5. Colorimetric Determination of MED with Mathematical Model and Comparing with Conventional Visual Method. The $a^{*}$ data of chromameter were mathematically modeled to assess the MED values. Objectively we proposed that the lowest subthreshold UV doses do not induce the erythema and, thus giving a horizontal line. At the threshold where UV begins to cause erythema (MED), a curve with a positive gradient would commence. The data were modeled to an initial horizontal line with a curve commencing at an unknown point (Figure 3) [7]. This unknown point, the intersection of the line and curve, was determined by mathematical modeling as the MED. Both average intercepts and slopes for each subject were calculated to see if a more objective measure of MED could be obtained.

The individual MED value evaluated by conventional visual determination and mathematical model is revealed at Figure 4. The average MED of all volunteers is 86.5 $\pm 22 \mathrm{mj} / \mathrm{cm}^{2}$ (change in $a^{*}$ of $+2.08 \pm 0.74$ units) by conventional visual method. The corresponding MED using mathematical model is $76.5 \pm 25 \mathrm{mj} / \mathrm{cm}^{2}$ (change in $a^{*}$ of $+1 \pm 0.77$ units). Using mathematical modeling, we are able to detect $a^{*}$ change in erythema at lower UV doses than the conventional visual assessment except case 14. Thus, we are $95 \%$ sure that MED determined by mathematical model is not equal to conventional visual method. However, the correlation of MED values between visual method and mathematical prediction is fair (Pearson correlation coefficient $=0.758)$.

\section{Discussion}

Appropriate instruments and mythology are indispensable to monitor the differences of epidermis response. The present study was designed to identify the discrimination capability among different noninvasive techniques. Previous studies have shown the ability of instruments such as the chromameter (CR) and the mexameter $\mathrm{Hb}$ to quantitate more sensitive measures of skin color changes [7]. From our results, colorimeric measurements (including mexameter and chromameter) and visual scoring give the highest discrimination of UVB irradiation, and $a^{*}$ and EI show a linear relationship to VS. Taken together, the reproducibility and convenience of $a^{*}$ is most satisfactory, even if three times of measurements were used. The $a^{*}$ provides reliable information and can be designed as in a mathematical model in predicting MED. Through detailed comparison of different UVB doses and MEDs, the parameters derived could be 
TABLE 1: The descriptive statistics of mean differences with biomedical techniques. EI, COR, and $a^{*}$ increase with higher UVB irradiation. On the contrary, MI, $L^{*}$, and $b^{*}$ decrease. Discrepancy: TEWL, COR, SC, DP, LDI.

\begin{tabular}{|c|c|c|c|c|c|}
\hline & Cases & Minimum & Maximum & Mean & SD \\
\hline MI100D & 20 & -28.00 & 12.00 & -8.2285 & 11.0630 \\
\hline MI200D & 20 & -75.00 & 2.00 & -45.6355 & 23.2291 \\
\hline EI100D & 20 & -10.75 & 92.00 & 47.5690 & 29.9449 \\
\hline EI200D & 20 & 36.00 & 299.00 & 154.9840 & 68.2407 \\
\hline TEWL100D & 20 & -2.20 & 6.00 & 0.7100 & 2.3799 \\
\hline TEWL200D & 20 & -6.40 & 3.20 & 0.1000 & 2.1866 \\
\hline COR100D & 20 & -10.00 & 23.89 & 2.4405 & 8.0193 \\
\hline COR200D & 20 & -11.20 & 35.56 & 1.2560 & 10.2402 \\
\hline SC100D & 20 & -6.00 & 13.00 & 0.8971 & 4.0347 \\
\hline SC200D & 20 & -4.34 & 7.00 & 0.8825 & 3.4885 \\
\hline DP100D & 20 & -13.00 & 26.00 & 2.5990 & 10.2211 \\
\hline DP200D & 20 & -15.00 & 21.00 & 1.4970 & 9.9801 \\
\hline LDI100D & 20 & -20.80 & 18.80 & -0.2200 & 11.6452 \\
\hline LDI200D & 20 & -7.00 & 625.00 & 213.7200 & 177.6635 \\
\hline L100D & 20 & -4.41 & 0.56 & -1.2520 & 1.1509 \\
\hline L200D & 20 & -6.77 & -0.25 & -3.1450 & 1.8638 \\
\hline A100D & 20 & 0.21 & 5.09 & 1.6305 & 1.1571 \\
\hline A200D & 20 & 1.11 & 9.57 & 4.6560 & 2.2557 \\
\hline B100D & 20 & -0.96 & 0.35 & -0.1850 & 0.3261 \\
\hline B200D & 20 & -1.46 & 0.77 & -0.5840 & 0.5619 \\
\hline VS100D & 20 & 0.00 & 2.00 & 0.7000 & 0.5712 \\
\hline VS200D & 20 & 1.00 & 3.00 & 2.2500 & 0.7864 \\
\hline
\end{tabular}

MI: mexameter melanin index; EI: mexameter erythema index; TEWL: transepidermal water loss; COR: corneometer CM 825; SC, thickness of stratum corneum; DP, minimal thickness of the epidermis; L, A, and B: colorimetric $L^{*}, a^{*}$ and $b^{*}$ measurements using Chromameter CR 400; LDI: laser-Doppler perfusion imaging; VS: visual score.

\#100D: Mean differences of \# measure at $100 \mathrm{mj} / \mathrm{cm}^{2}$ UVB-irradiated doses.

\#200D: Mean differences of \# measure at $200 \mathrm{mj} / \mathrm{cm}^{2}$ UVB-irradiated doses.

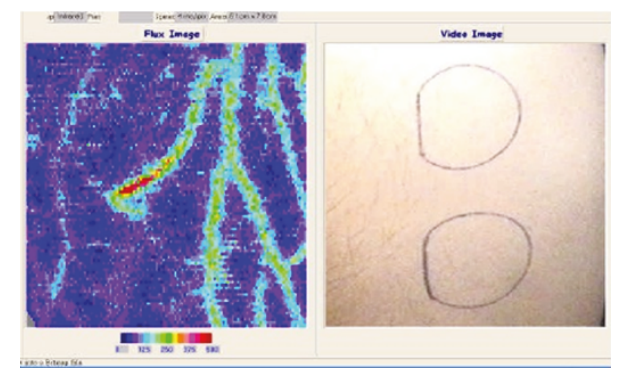

(a)

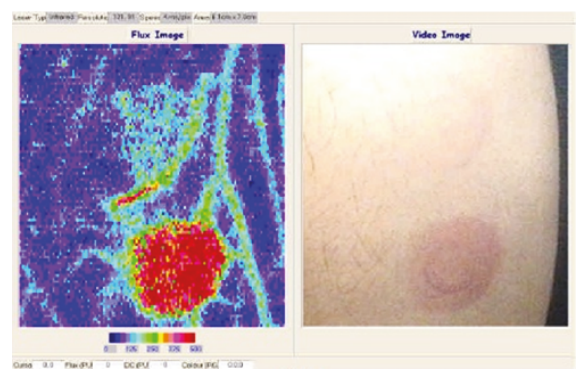

(b)

FIGURE 2: The LDI range features a CCD camera which produces a color image of the scanned area, making the positioning and comparison of images easier. (a) Before UVB irradiation. (b) After 100 and $200 \mathrm{mj} / \mathrm{cm}^{2}$ UVB-irradiation.

applied in photobiologic studies. Besides, standardization of the method developed in this present study may have defense implication in the future.

Olson et al. reported that MED correlated well with melanosome size, quantity, density, and distribution in various skin colors [14]. Lee et al. showed that hyperkeratosis and acanthosis were more prominent $24-48$ hours after single dose of 2 MED UVB. Marked hyperkeratosis was observed in about $20 \%$ after 24 hours [11]. However, we found no dosedependent effect and no obvious discrimination in terms of stratum corneum thickness. Possibly, any minor movement in our subjects may interfere with delicate measurements of CLSM. Besides, the brightness decreased after UVB irradiation. Compared with LSCM analysis, temporary decrease of brightness may be due to rapid proliferation of keratinocytes rich in supranuclear melanin caps after UV exposure and upward movement, resulting in darken epidermal basal layer. Further examination of images from stratum corneum to the epidermal-dermal junction could be performed by image processing. 


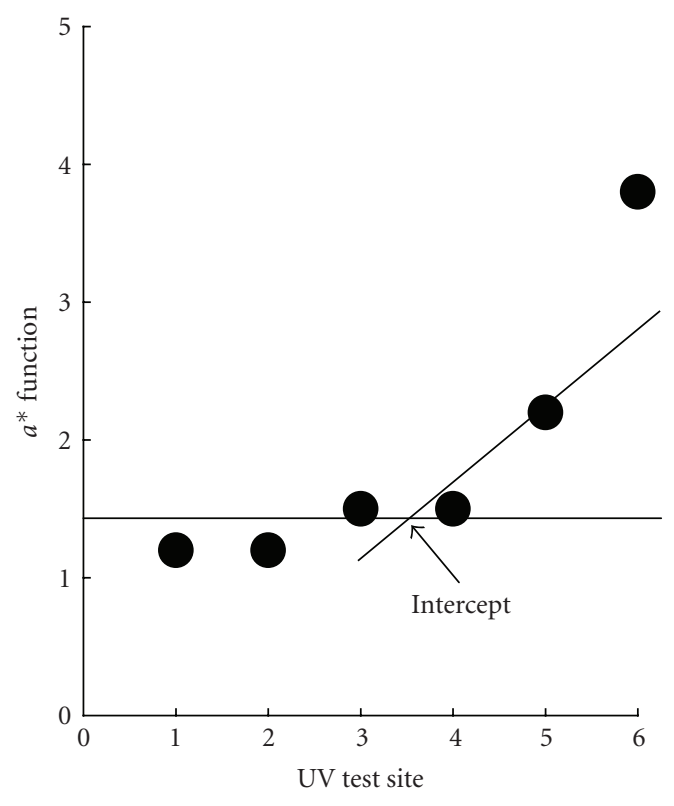

FIGURe 3: Colorimetric determination of MED with mathematical model.

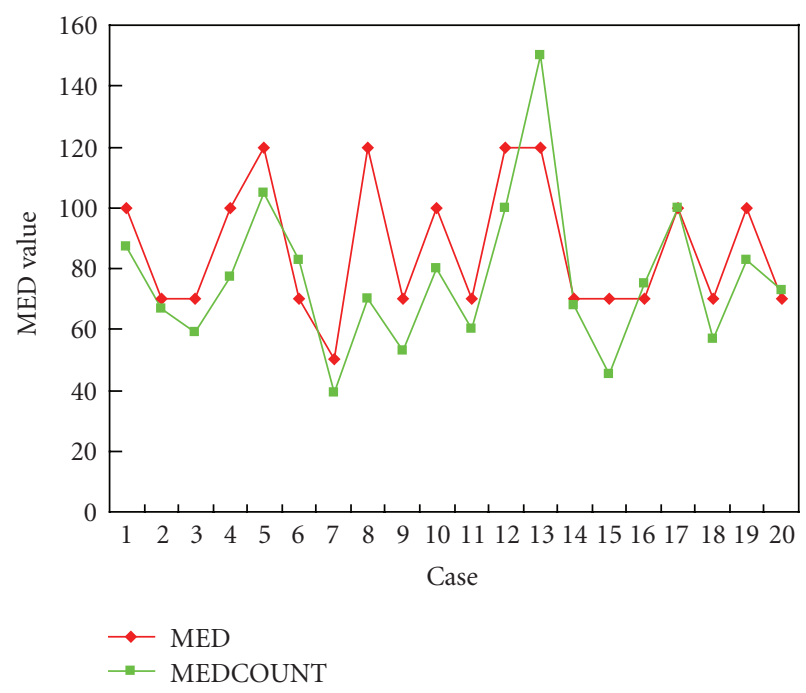

FIgUre 4: The individual MED value evaluated by conventional visual determination and mathematical model. MED: minimal erythema dose from visual assessment. MEDCOUNT: MED counted by mathematical model.

By using LDI, we also found positive relationship between blood flow of skin and UVB irradiation. It appears that UVB irradiation can enhance the dermal microperfusion, as with inflammation and vasodilation of skin induced by UV exposure [4].

Transepidermal water loss (TEWL) is a well-documented method for studying the skin barrier function through evaporation changes $[15,16]$. However, these changes are partly due to environmental factors as well as the psychological/ physiological status of the person tested. The bioengineering methods of this study could not discriminate the effects of UVB on barrier function and water content of skin. Schempp el al. demonstrated that exposure to $5 \%$ saltwater leads to a decrease of threshold for elicitation of UVB-induced erythema with an increase of erythemal response $[17,18]$. To clarify the correlation between water content and UVB irradiation, no discrimination was demonstrated between the measures and mean difference of corneometer, implying that water content and barrier function are not correlated with UVB irradiation. Besides, barrier disruption should be interpreted with caution, as a decrease is seen initially. Indeed, Fluhr el al. mentioned that barrier damage is a late effect of acute UV irradiation. Attention can also be detected using VS during early phase (until 48 hours) [12].

Consistent with Westerhof et al. [19], we found no significant correlation between MEDs and constitutive colorimetric readings. The MED is only an estimate of amount of UV radiation required for erythema. We cannot simply predict the MED value from constitutive colorimetric readings, because no correlation was observed between UV sensitivity and skin color.

Dose-response data of erythema more accurately measure the responses of human skin to UVB. A sophisticated chromameter supported by mathematical modeling will offer objective measurement of erythema to UV radiation and dose-response relationship.

When plotting erythema curves (as measured by $a^{*}$ ) for each volunteer, each curve seems to be the composite of two components-a horizontal component without measurement of erythema and a second curve containing measurable erythema. The mathematical modeling we proposed can identify these two curves, their intercept and the slope of the second curve. We proposed that the intercept represents the threshold where biological change in erythema occurs. Theoretically, the MED predicting by mathematical model is lower than visual assessment $\left(10 \mathrm{mj} / \mathrm{cm}^{2}\right.$ in our study). Using mathematical modeling, we are able to detect $a^{*}$ change in erythema at lower UV doses than the conventional visual assessment. Whereas, the reason why the 14th case in Figure 4 shows higher counting MED value may be due to only 6 plottings in the phototests. We suspect that more UVB-irradiated plottings would be better to determine the more accurate intercept in future studies. In addition, whether the slope is a useful method in differentiation between different skin types compared with visual MED deserved further investigation.

Diverse skin responses need different measurement modalities to achieve a satisfactory discrimination. It is more desirable to have objective measurement in the assessment of UV damage, such as MED. The proposed mathematical model using intercept predicted by chromameter $a^{*}$ function may be a supplementary method in the measurement of MED. With this approach, we are able to monitor the changes of erythema after UVB irradiation. The assessment of sensitivity of human skin to UV radiation is important for military personnel movement that is exposed to sun and sunburn is a major problem. This study identifies a noninvasive parameter in the evaluation of in-vivo dose and epidermis changes following UVB irradiation. A more objective MED is of great help for sunburn risk screening and prevention. 


\section{Acknowledgment}

This research was supported by the Veterans General Hospital-Kaohsiung, Grant no. VGHKS 96-87.

\section{References}

[1] I. E. Kochevar and C. R. Taylor, "Photophysics, photochemistry and photobiology," in Fitzpatrick's Dermatologhy in General Medicine, I. M. Freedberg, A. Z. Eisen, K. Wolff, et al., Eds., pp. 1267-1275, McGraw-Hill, New York, NY, USA, 6th edition, 2003.

[2] J. Krutmann, H. Honigsmann, C. A. Elmets, and P. R. Bergstresser, "Dermatological phototherapy and photodiagnostic methods," in The Photopatch Test, pp. 338-341, Springer, Berlin, Germany, 2001.

[3] http://www.konicaminolta.com/sensingusa/products/color/ colorimeters/cr400-410/index.html.

[4] M. A. Allias, K. Wårdell, M. Stücker, C. Anderson, and E. G. Salerud, "Assessment of pigmented skin lesions in terms of blood perfusion estimates," Skin Research and Technology, vol. 10, no. 1, pp. 43-49, 2004.

[5] C. K. Kraemer, D. B. Menegon, and T. F. Cestari, "Determination of the minimal phototoxic dose and colorimetry in psoralen plus ultraviolet A radiation therapy," Photodermatology Photoimmunology \& Photomedicine, vol. 21, no. 5, pp. 242248, 2005.

[6] http://www.courage-khazaka.de/.

[7] T. S. C. Poon, J. M. Kuchel, A. Badruddin, et al., "Objective measurement of minimal erythema and melanogenic doses using natural and solar-simulated light," Photochemistry and Photobiology, vol. 78, no. 4, pp. 331-336, 2003.

[8] K. Sauermann, S. Clemann, S. Jaspers, et al., "Age related changes of human skin investigated with histometric measurements by confocal laser scanning microscopy in vivo," Skin Research and Technology, vol. 8, no. 1, pp. 52-56, 2002.

[9] S. Nouveau-Richard, M. Monot, P. Bastien, and O. de Lacharrière, "In vivo epidermal thickness measurement: ultrasound vs. confocal imaging," Skin Research and Technology, vol. 10, no. 2, pp. 136-140, 2004.

[10] http://vivascopy.com/medical-imagers/vivascope-1500.asp.

[11] T. Gambichler, K. Sauermann, M. A. Altintas, et al., "Effects of repeated sunbed exposures on the human skin. In vivo measurements with confocal microscopy," Photodermatology Photoimmunology \& Photomedicine, vol. 20, no. 1, pp. 27-32, 2004.

[12] J. W. Fluhr, O. Kuss, T. Diepgen, et al., "Testing for irritation with a multifactorial approach: comparison of eight noninvasive measuring techniques on five different irritation types," British Journal of Dermatology, vol. 145, no. 5, pp. 696703, 2001.

[13] B. L. Diffey, C. T. Jansen, F. Urbach, and H. C. Wulf, "The standard erythema dose: a new photobiological concept," Photodermatology Photoimmunology \& Photomedicine, vol. 13, no. 1-2, pp. 64-66, 1997.

[14] R. L. Olson, J. Gaylor, and M. A. Everett, "Skin color, melanin, and erythema," Archives of Dermatology, vol. 108, no. 4, pp. 541-544, 1973.

[15] H. Miyauchi, T. Horio, and Y. Asada, "The effect of ultraviolet radiation on the water-reservoir functions of the stratum corneum," Photodermatology Photoimmunology \& Photomedicine, vol. 9, no. 5, pp. 193-197, 1992.

[16] J. J. Thiele, F. Dreher, H. I. Maibach, and L. Packer, "Impact of ultraviolet radiation and ozone on the transepidermal water loss as a function of skin temperature in hairless mice," Skin Pharmacology and Applied Skin Physiology, vol. 16, no. 5, pp. 283-290, 2003.

[17] M. Moehrle, W. Koehle, K. Dietz, and G. Lischka, "Reduction of minimal erythema dose by sweating," Photodermatology Photoimmunology \& Photomedicine, vol. 16, no. 6, pp. 260262, 2000.

[18] C. M. Schempp, K. Müller, J. Schulte-Mönting, E. Schöpf, and J. C. Simon, "Salt water bathing prior to UVB irradiation leads to a decrease of the minimal erythema dose and an increased erythema index without affecting skin pigmentation," Photochemistry and Photobiology, vol. 69, no. 3, pp. 341-344, 1999.

[19] W. Westerhof, O. Estevez-Uscanga, J. Meens, A. Kammeyer, M. Durocq, and I. Cario, "The relation between constitutional skin color and photosensitivity estimated from UV-induced erythema and pigmentation dose-response curves," Journal of Investigative Dermatology, vol. 94, no. 6, pp. 812-816, 1990. 